\title{
OBJECT DETECTION AND IDENTIFICATION BY HIERARCHICAL SEGMENTATION
}

\author{
Patrick HANUSSE and Philippe GUILLATAUD \\ Centre de Recherche Paul Pascal, CNRS \\ Château Brivazac, F-33600 PESSAC
}

One of the traditional problems of vision is to understand the devices of the tremendous compression of information that is performed so rapidly and apparently so easily by human vision. To extract meaning from numbers, hierarchical analysis is a possible answer. It has been used in many contexts as a way to deal with complex situations and systems $[1,2]$. Its most important feature resides in its factorization property, which recursively splits difficult tasks into simpler ones. To do so, one should be able to design an operational procedure to construct some meaningful hierarchical structure from a complex observation.

To avoid ambiguity and undesirable references to broad, fuzzy or extensively used terminology, and to stress its quite universal status, we define as "dendronic analysis" a general method that is able to produce a significance coding tree structure from a set of measurements of any kind, an a "dendrone" or "dendronic structure", the tree structure that is produced.

We will deal here with segmentation as an object self-detecting method, i.e. that extracts objects without requiring any a priori knowledge of the objects, an idea that we have already explored [3].

\section{Definition of dendronic analysis}

We shall consider thresholding as a process in which segmented regions are less important than the way they change with threshold value. We are more interested in the qualitative changes, which are structural changes, rather than in quantitative ones.

Considering the picture intensity profile as a seascape and the threshold value as sea level, we initially start with the sea covering everything. We let the sea level go down, and we record the position and tide level of appearing islands. From one tide level to the other, three events may occur: (i) a new island appears, (ii) an existing island grows in area, (iii) two nearby separated islands merge into a larger one. From a strictly structural point of view, the last event is the most important. It defines a branching process, a qualitative change. The structure of the collected information is one of a tree, the nodes of which may be labelled by various types of information. In this process a large amount of less pertinent information is left out. This information is of morphological nature. The dendronic analysis aims at separating topology from morphology.

\section{Properties of dendronic analysis}

Discretization effects: There are two effects due to the discrete nature of a digital picture, in space and amplitude, but also to physical cutoffs related to noise amplitude and correlation. They can be controlled by two parameters of the analysis, namely, the Minimum Island Size (MIS), and the Sea Level Decrement (SLD). The MIS defines the smallest island area that is detectable and can be added to the dendrone. The SLD defines the intensity resolution, and controls the local contrast that is required to form an island.

Filtering properties: These very same parameters can also have a role at picture comprehension level as they may be used to specify a possible a priori knowledge of minimum object size and contrast. Consequently, they act as filtering parameters in the dendronic analysis itself. Filtering could act on the dendrone itself, to rednce its complexity and increase its significance. In this case we would speak of dendrone driven pattern recognition. 
The efficiency of pattern recognition controlled by the dendronic structure is increased by its higher significance content as compared to the original picture or region and contour segmentation.

Adaptability and Invariance properties: The topology of a dendrone is not dependent on local intensity levels, so that two objects with the same structure located in different lightening or contrast conditions will appear with the same, simply shifted, dendronic contribution. The method is thus naturally adaptative.

It is also easy to show that the same structural topology will be invariant under translation, rotation, and scaling. This is again a consequence, and benefit, of the separation between the structural content of a picture and any local contextual dependence on lightening, position, orientation and magnification.

Robustness: Its adaptative character is already providing robustness to long range defects. The dendronic contribution of noise is unambiguously detectable. It can be eliminated by adjusting the two already mentioned parameters, MIS and SLD.

Finally, another reason for robustness is due to the structural stability of the topology of a picture. The dendronic structure emphasizes the mutual topological relationship between regions, rather than the regions themselves. This is not much perturbated by random noise or context variations.

Significance extraction: Filtering processes acting on a dendrone increase its significance. But, the most important aspect of dendronic analysis resides certainly in self-building significance. By this, we mean that, besides being adaptative and robust, this method reveals the internal segmentation, or the hierarchical levels of description, that are present in the picture, without having to formulate explicitly a model of the scene and of the objects it contains. It is easy to setup a numerical discrimination criterion on the dendrone itself that would implement rules such as "noise branches at all levels, objects don't". This is what we call significance self-detection.

\section{First example: Disks (Fig. 1)}

This noisy artificial picture (256x256) represents two disks on a non uniform background (fig.1a). Figure $1 \mathrm{~b}$ displays a diagonal cross-section showing backgound slope and disk amplitude which is lower than noise level. This noise is spatially correlated over 5 pixels. This is a very difficult situation for thresholding or any region or contour segmentation. Figure $1 \mathrm{~d}$ presents a representation of the dendrone (MIS $=100, \mathrm{SLD}=2$ ) with node horizontal base size proportional to region area. Dendrone structure inspection, along with several quantitative parameters collected during dendronic analysis, allows us to separate unambiguously, at two different levels, the two objects present in the picture (see thick lines in fig 1d). This is what we call self-detection. Figure 1c gives the two extracted disks at their own self-determined levels (not a plain picture thresholding). This example shows the robustness and adaptativity of the method.

\section{Second example: Bolts (Fig. 2)}

A group of 9 bolts is set on a more or less uniform background (Fig 2a). Lightening conditions are not very good. There are shadows between the objects, which would make it difficult to find a good segmentation threshold. A global view of the corresponding dendrone (half of it) is given in fig. 2b. The representation uses the base area has horizontal scale. Five bolts are very easily detectable. The varying local base threshold reveals again adaptativity. It is noticeable that there is a structure within the bolts, and that this structure can be found in each bolt. Fig. 2c presents two magnified objects dendrones, here using plain structure representation (horizontal scale as no physical meaning). The bolt head groups (H1,H3) and thread pairs (T1,T3) can be identified. This structure is invariant under translation and rotation. It would also be invariant under scaling. Getting rid of these possible sources of contextual dependences obviously greatly simplifies the identification step. 


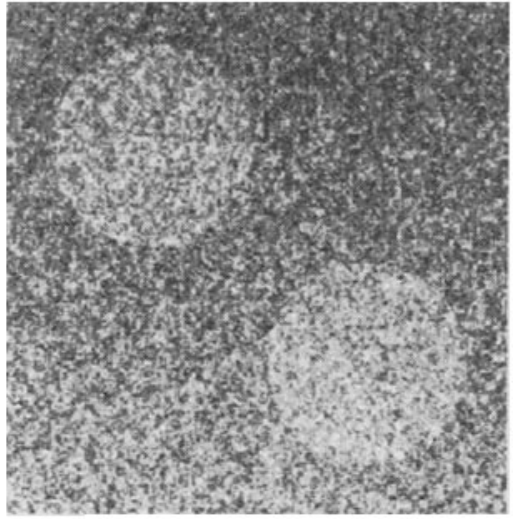

(a)

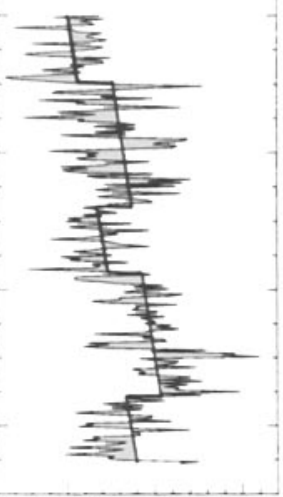

(b)

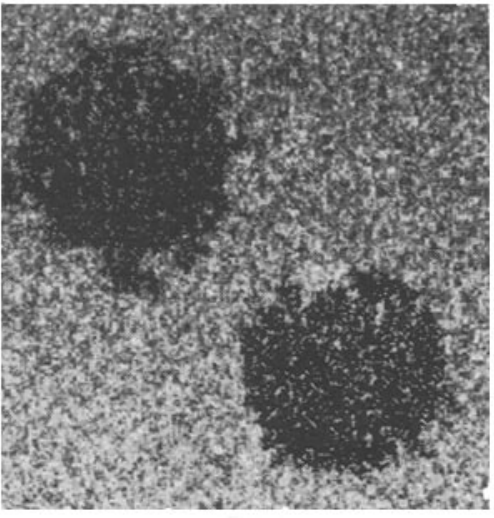

(c)

Fig 1: Disks

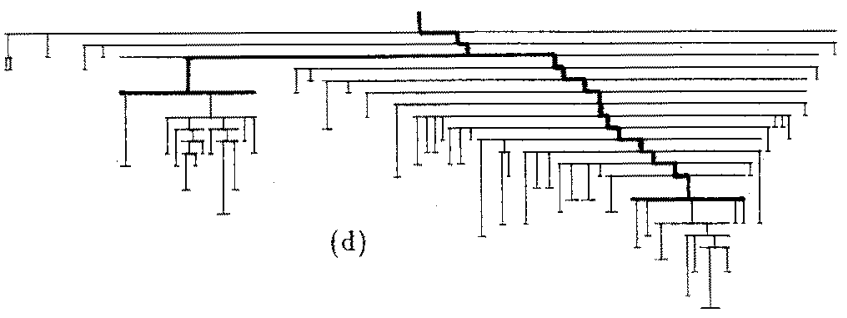

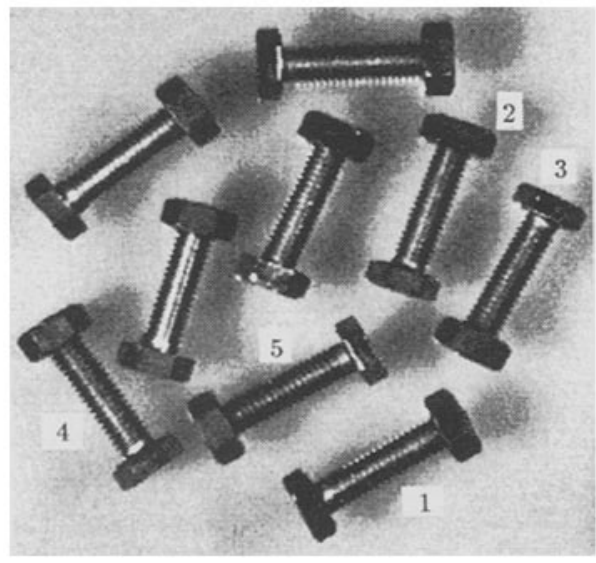

(a)

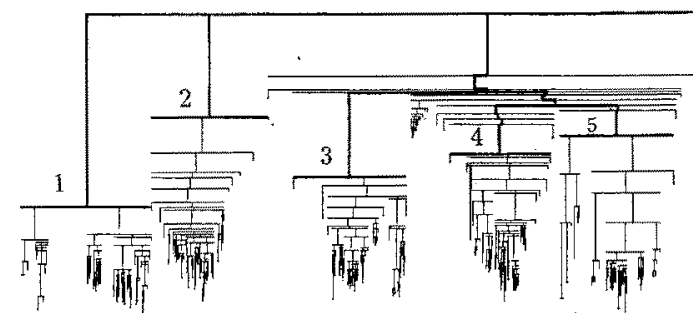

(b)

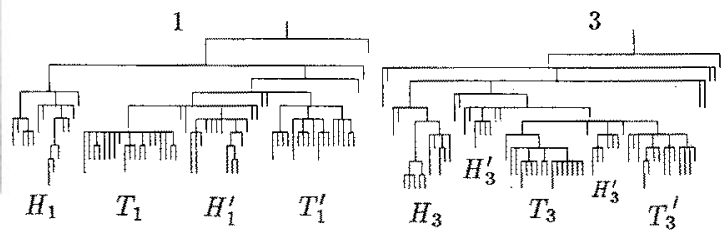

(c)

Fig 2: Bolts

\section{References}

[1] R. DUDA and P. HART, "Pattern classification and scene analysis", Wiley (1973).

[2] H. SAMET and R.E. WEBBER, "Hierarchical data structures. A review". Proceeding of the International Electronic Image Week, Nice, April 1986, CESTA Paris, (1987) and references therein. [3] P. HANUSSE, "Analyse morphologique de trajectoires en dynamique chimique" J.Chim.Phys. $84,1315-1327,(1987)$. 